\title{
Carriage of Candida species and $C$ albicans biotypes in patients undergoing chemotherapy or bone marrow transplantation for haematological disease
}

\author{
F C ODDS, C C KIBBLER, * E WALKER, $\dagger$ A BHAMRA, * H G PRENTICE, $\dagger$ P NOONE*
}

From the Department of Microbiology, University of Leicester, the *Departments of Medical Microbiology and $\dagger$ Academic Department of Haematology, Royal Free Hospital, London

SUMMARY Six hundred and seventy four yeast isolates obtained from routine microbiological screening of 153 patients with haematological disease were identified and Candida albicans isolates biotyped over nine months to determine longitudinal and cross sectional patterns of yeast colonisation. A yeast microflora persisted in many patients despite the routine prophylactic use of oral antifungal agents. Analysis of the yeast species isolated on a cross sectional basis showed that $C$ albicans accounted for $65 \%$ of yeasts isolated from the oral cavity but only $45 \%$ of the faecal yeast flora. Longitudinal changes in yeast flora occurred significantly more often in faecal samples than in oral samples and significantly less often in sites colonised with $C$ albicans than in sites colonised with other species. No associations were found between the yeasts isolated and the nature of antifungal prophylaxis used, or the extent of a patient's stay in hospital.

Patients with leukaemia and other forms of haematological malignancy comprise a group at high risk for systemic forms of candidosis. ${ }^{1}$ While many surveys have examined the prevalence and prevention of clinically important Candida infection in such patients, very few have studied their yeast flora in the absence of overt fungal infection. Most studies of this type have involved patient populations with a wide spectrum of malignant diseases. Only Tomoda et al specifically surveyed patients receiving anti-leukaemia chemotherapy $y^{2}$ : they found higher faecal Candida concentrations among 56 such patients compared with healthy controls and identified the Candida species in 29 isolates.

This study was designed to examine the Candida species and $C$ albicans biotypes isolated in routine samples from patients undergoing chemotherapy or bone marrow transplantation for haematological disease. By identifying and biotyping all yeast isolates received from such patients, it was hoped to determine longitudinal as well as cross sectional patterns of yeast colonisation in this particular group of patients.

\section{Patients and methods}

The study group comprised 153 patients nursed in either a general ward, a 28 bed mixed haematology/

Accepted for publication 28 July 1989 chest diseases ward, or a general paediatrics ward. About $40 \%$ of treatment courses were undertaken in single rooms using protective isolation techniques. The period of the study spanned October 1985 to July 1986. The patients had various haematological malignancies, predominantly acute myeloblastic leukaemia, and were receiving induction/remission chemotherapy according to several different protocols. Nineteen patients underwent allogeneic bone marrow transplantation for acute leukaemia, chronic granulocytic leukaemia, lymphoma or aplastic anaemia, and four patients had autografts for acute lymphoblastic leukaemia.

Almost all patients received antifungal prophylaxis during their hospital stay with oral amphotericin B 500 $\mathrm{mg}$ four times a day and ketoconazole $200 \mathrm{mg}$ twice a day. Ten patients received ketoconazole alone and seven were given itraconazole $100 \mathrm{mg}$ daily. Nine patients received no antifungal prophylaxis. All patients were given colistin $\left(1.5 \times 10^{6}\right.$ units 12 hourly by mouth), neomycin ( $500 \mathrm{mg} 12$ hourly by mouth), and $1 \%$ povidone iodine mouth washes $(5 \mathrm{ml}$ six hourly).

Routine microbiological screening entailed regular culture of oral or throat swabs and faeces on Sabouraud's glucose agar as well as on bacteriological culture media.

All cultures positive for yeast were stored at $4^{\circ} \mathrm{C}$ for two to eight weeks before they were processed for yeast 
identification and biotyping. The protocol for identification and biotyping of yeast isolates was designed to maximise the probability of detection of instances where multiple yeast types occurred in a single sample. Three colonies were selected from each culture plate. When different colonial forms (determined by their size, hue, texture and morphological detail) were seen on the plate, examples of each form were included among the three selected. Where the colony characteristics seemed to be identical, three colonies were selected at random.

The triplicate isolates from each clinical sample were processed for presumptive species identification and for differentiation of $C$ albicans biotypes in physiological tests. ${ }^{3}$ Isolates were tested for growth at $\mathrm{pH} 1 \cdot 55$, resistance to cetrimide, and lack of growth on Difco MacConkey's agar to establish their presumptive identity as $C$ albicans. $C$ albicans isolates were further typed according to their ability to grow at $\mathrm{pH}$ 1.40 , production of clear zones in protein agar, resistance to flucytosine, boric acid, and safranine, salt tolerance, and utilisation of urea, sorbose, and citrate. All isolates giving negative or equivocal results in the presumptive tests for identification of $C$ albicans were identified on the basis of their microscopic morphology and their profiles in API 20C tests, direct pour-plate auxanograms, ${ }^{5}$ or both. Individual $C$ albicans clones from a single clinical specimen were recorded as different biotypes when their biotype patterns were disparate in two or more tests.

For convenience, individual biotype patterns of $C$ albicans isolates were referred to by means of three digit codes. Each digit uniquely encodes the results of three of the nine tests where a positive result is scored 1,2 , or 4 , and the digit represents the total score for these tests. For example, a result of ++--++ --+ would be encoded as 364 .

The biotyping tests were conducted entirely without any knowledge of the identity of the patients from whom the samples came. When the blind testing was completed, the results were matched to their sources. Where sequential samples from the same patient had been typed, the biotype pattern was similar throughout but with small variations. For example, in one patient from whom 13 sequential samples yielded $C$ albicans, the biotypes found were $153(\mathrm{n}=8), 157(\mathrm{n}=$ $3)$, and $053(n=2)$. As the biotyping system has been shown to possess a batch-to-batch reproducibility of only one or two test differences ${ }^{3}$ and as, in most cases, the results were obtained from many separate test batches, it was decided that interpretation of the above results as temporal variations in biotype was invalid in the longitudinal studies. For these studies alone, a computerised iterative procedure was used for each set of multiple sample results from a single patient to determine a "consensus" biotype pattern and to detect outlying (truly different) types. This procedure involved determining the most common pattern of positive and negative test results from the biotype data, then determining which individual isolates gave patterns that differed from this "majority" type by more than two test differences. The latter types were then resubjected to the pattern determination procedure. In the example given above, the "consensus" biotype for all the isolates was 153 .

\section{Results}

A total of 674 cultures positive for yeast was received from 153 patients with leukaemia during the study period. Most isolates were from the oral cavity and from faeces. The remainder were from miscellaneous sources, particularly sputum, the vagina, the nose and perineal swabs. Seven isolates were from the tips of central venous catheters, three came from cerebrospinal fluid, and one each came from a blood culture and a biopsy specimen. The number of isolates from putatively deep seated sites was therefore considered to be too small to permit statistically valid interpretation. At least three isolates were obtained from 45 of the patients.

Isolates from both the mouth and the faeces of an individual patient were obtained in 57 instances on the same date. In 35 of these instances the Candida species or $C$ albicans biotype was the same in both specimens; in 18 the Candida species isolated were different, and in four the $C$ albicans biotypes isolated differed between the oral and faecal isolates.

Table 1 No (\%) of yeast species and C albicans biotypes in different types of sample from patients with haematological disease

\begin{tabular}{lllllll}
\hline \multirow{2}{*}{$\begin{array}{l}\text { Sample source } \\
\text { (No of isolates) }\end{array}$} & \multicolumn{2}{l}{ Calbicans biotype } & & \multicolumn{2}{c}{ C } \\
\cline { 2 - 6 } & $1--$ & $3--$ & Other & C glabrata & C tropicalis & Other spp \\
\hline Oral cavity $(\mathrm{n}=84)$ & $17(20)$ & $23(27)$ & $14(17)$ & $8(10)$ & $5(6)$ & $17(20)$ \\
Stools $(\mathrm{n}=82)$ & $9(11)$ & $10(12)$ & $18(22)$ & $17(21)$ & $5(6)$ & $23(28)$ \\
Other sources $(\mathrm{n}=91)$ & $16(18)$ & $20(22)$ & $19(21)$ & $14(15)$ & $5(5)$ & $17(19)$
\end{tabular}

Biotype group 1-- includes isolates that were proteinase negative but $\mathrm{pH} 1.4$ positive; biotype group 3- - includes isolates that were positive in both these tests but sensitive to flucytosine. 
Table 2 Incidence of $C$ albicans biotypes in different types of sample from patients with haematological disease

\begin{tabular}{llll}
\hline $\begin{array}{l}\text { No of } \\
\text { occurrences }\end{array}$ & $\begin{array}{l}\text { Biotypes in oral } \\
\text { cavity samples }\end{array}$ & $\begin{array}{l}\text { Biotypes in stool } \\
\text { samples }\end{array}$ & $\begin{array}{l}\text { Biotypes in } \\
\text { other samples }\end{array}$ \\
\hline 1 & $001,004,011,015,026,033,035,053$, & $001,003,013,016,031,033,044,071$, & $003,013,023,031,033,055,057$, \\
& $113,115,146,171,177,251,311,315$, & $077,113,171,210,211,213,251,253$, & $077,113,131,133,201,213,255$, \\
& $317,337,340,345,357,371,401,557$, & $313,317,345,347,350,351,557$ & $301,313,350,355,400,451,777$ \\
2 & 713 & 117,153 & $177,317,350,351,357,377,557$ \\
3 & $151,153,215,355$ & 157 & 153,214 \\
4 & 117 & 055,353 & 157,353 \\
5 & 313 & & \\
8 & 157 & & \\
9 & 353 & & \\
\hline
\end{tabular}

CROSS SECTIONAL SURVEY OF CANDIDA ISOLATES FROM PATIENTS WITH HAEMATOLOGICAL

\section{DISEASE}

Tables 1 and 2 summarise the numbers of Candida species and $C$ albicans biotypes isolated in a cross sectional survey of the study group. For these compilations, only the first sample isolated from a particular source in any patient was included, so that no patient/ sample combination is represented more than once. $C$ albicans was the most common species found from all sites (table 1), but other species, notably $C$ glabrata and Saccharomyces cerevisiae, were isolated reasonably often from faecal samples. The proportion of species other than $C$ albicans isolated from faeces was significantly greater than that from oral samples and other sources $\left(\chi^{2}\right.$ test, $\left.\mathrm{p}<0.05\right)$. The $C$ albicans biotypes isolated were mainly those with codes $1--$ or 3--; respectively, types that were $\mathrm{pH} \mathrm{1.4}$ positive, proteinase negative, and $\mathrm{pH} 1.4$ positive, proteinase positive (tables 1 and 2). Types 157 and 353 were the most common.

Mixtures of Candida species or $C$ albicans biotypes were obtained in 25 of the samples. Six of the oral samples contained $C$ albicans plus another Candida sp; one contained $C$ glabrata plus $C$ parapsilosis; and one contained $C$ albicans plus $S$ cerevisiae. Two oral samples contained two $C$ albicans biotypes. Among the faecal samples, $C$ albicans plus another Candida sp was found on six occasions, $C$ albicans plus $S$ cerevisiae once, and two $C$ albicans biotypes once. The remaining instances of mixed populations occurred in two samples of sputum, a vaginal swab, a nasal swab, a perineal swab and an eye sample.

\section{YEAST FLORA IN INDIVIDUAL PATIENTS}

Table 3 gives details of isolates obtained in 12 patients from whom at least 12 yeast positive samples were obtained. Except where stated below, all 12 patients received prophylactic cover with amphotericin $B$ plus ketoconazole. The results are presented in detail for these patients because they convey more clearly than any analysis the often complex nature of the findings of this survey. They show that for most patients a consistent yeast microflora was harboured at a given site. Case 1 who received no antifungal prophylaxis, was consistently colonised with $C$ tropicalis, except for a single occasion when $C$ glabrata was isolated from a stool sample. Case 2 was colonised orally and faecally mainly with $C$ albicans biotypes 015 and 215 (consensus biotype 215) on three separate admissions to the ward. $C$ tropicalis was also isolated frequently from oral samples during the first admission, and $C$ glabrata was present in several faecal samples during admissions 1 and 3.

Case 3 was colonised with Candida mainly in faecal samples on three separate admissions. $C$ glabrata was the species isolated most often, but $C$ krusei was encountered in some oral samples during the first two admissions, and $C$ albicans was isolated from single oral and faecal samples during the first admission. This patient received ketoconazole alone for antifungal prophylaxis but amphotericin B was added in subsequent admissions. Case 4 was also colonised with Candida mainly in faecal samples, but the flora was complex. A fiora of Calbicans (consensus biotype 055) was present during the first three weeks of this patient's first admission, but this species was replaced first with a mixed flora of $C$ krusei and $S$ cerevisiae, then with $C$ glabrata and $S$ cerevisiae, a combination that persisted through the patient's second and third hospital admissions.

Case 5 was positive for Candida primarily in the oral cavity, and his $C$ albicans flora persisted through five hospital admissions. The $C$ albicans biotype patterns varied from sample to sample in this patient, but all isolates were within two test differences of the consensus type 353. Case 6 was colonised with $C$ albicans consensus biotype 345 in oral and faecal samples during three admissions. Her prophylactic antifungal treatment varied between admissions: itraconazole was used alone during the first admission; amphotericin B plus ketoconazole in subsequent admissions. Case 7 similarly retained a $C$ albicans flora (consensus type 353) throughout four hospital admissions, although in this patient occasional faecal samples yielded $C$ glabrata during the first two admissions. 
Table 3 Details of yeasts isolated from patients for each week of their hospital stay

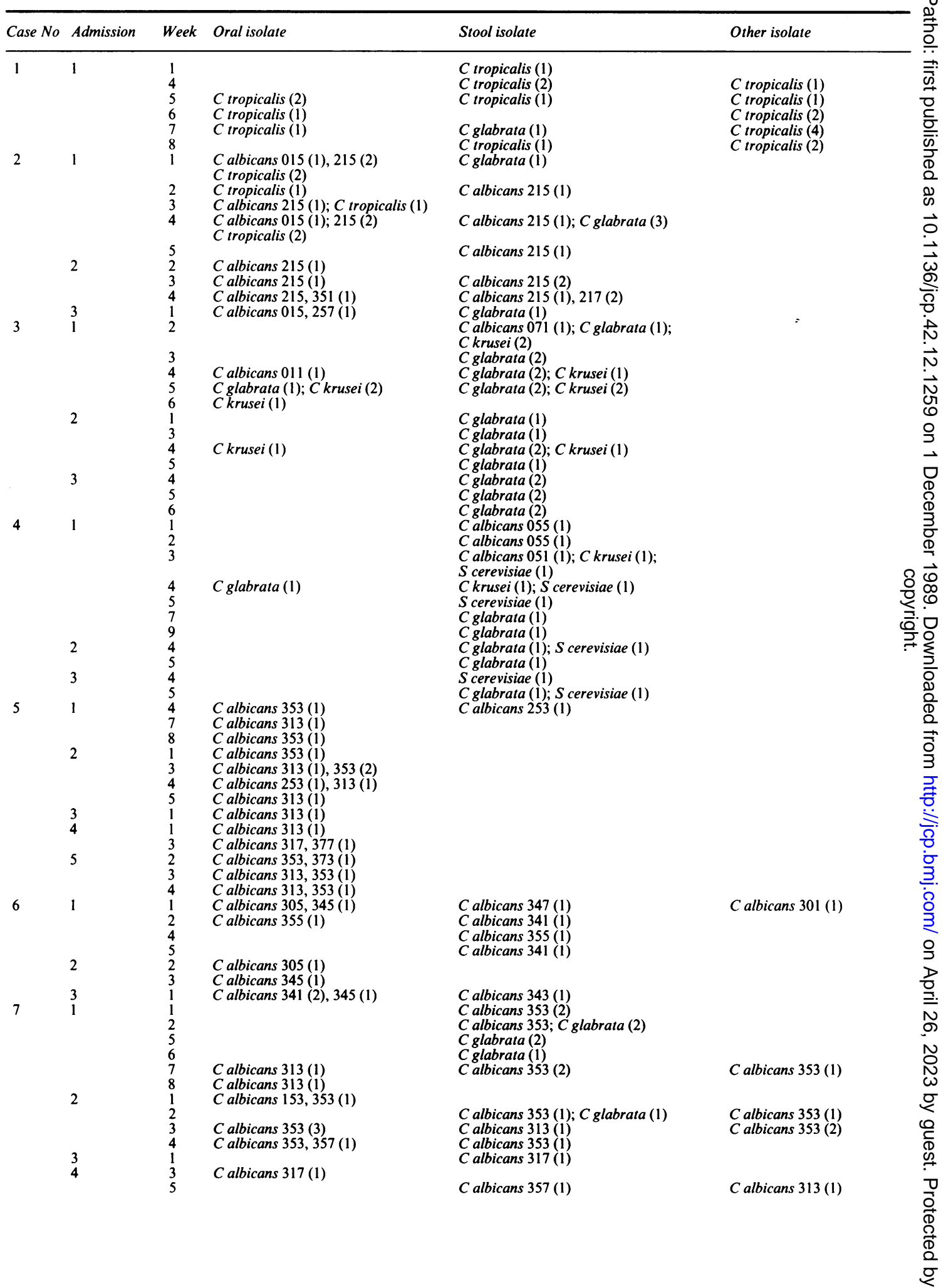


Table 3 continued

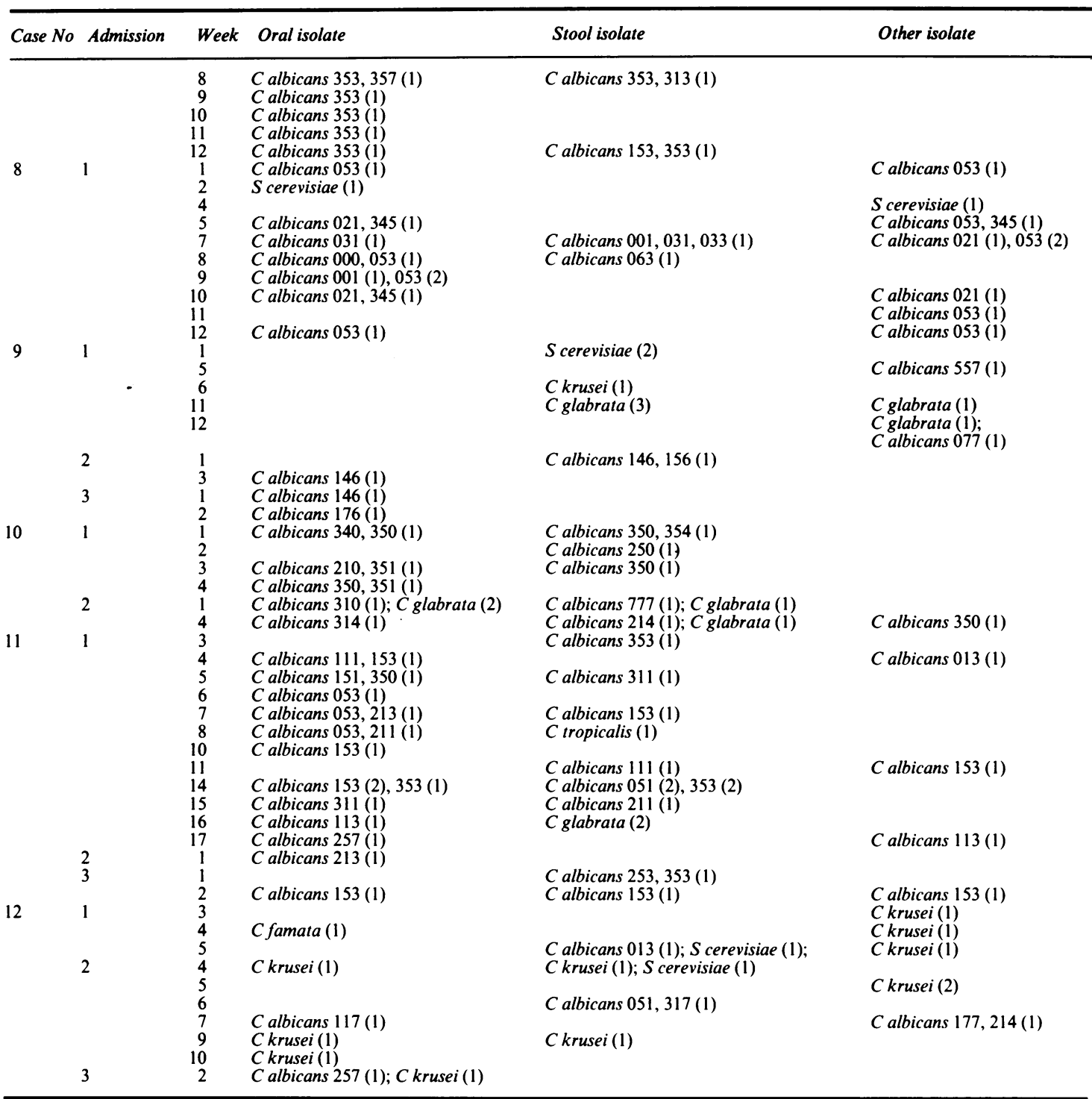

The 12 patients included in the table were those for whom 12 or more yeast isolations were recorded in the study. Numbers of occasions on which each species or $C$ albicans biotype were isolated are given in parentheses.

Case 8 is an example of a case in which the $C$ albicans biotypes isolated seemed to vary considerably (table 3). Computer analysis suggested there were, in fact, two main biotypes and one less common biotype in this patient. Types $000,001,021$ and 031 all fell within two test differences of consensus type 021 ; types 033 , 053, 063, 353 all fell within two test differences of consensus type 053 . The less common type was type 345 , which was the consensus biotype for types 345 and 357 . In addition to these three $C$ albicans types,
$S$ cerevisiae was recovered from one oral and one sputum specimen. Case 9 seems to have acquired a $C$ albicans flora between his first and second hospital admissions. The three different yeast species recovered from his faeces during the first admission were not isolated at all during admissions 2 and 3. C albicans types 557 and 077 , which were isolated from this patient's nose and perineum, respectively, during the first admission, bore no resemblance to the $C$ albicans consensus type 146 that was isolated repeatedly during 
admissions 2 and 3.

During her two admissions, Case 10 repeatedly yielded oral and faecal samples positive for $C$ albicans with a consensus biotype of 350 . During the second admission the new biotypes 214 and 777 were recovered from individual faecal samples and $C$ glabrata was isolated from faeces on two occasions. Case 11 was colonised with two repeatedly isolated $C$ albicans biotypes: 033, 051, 053, 111, 113, 151, 153, 253 and 353 suggest a consensus biotype of 153; types 211 , 213 , and 311 correspond to the consensus biotype 211 . Types 350 and 257 were also isolated once each from oral swabs, $C$ glabrata was isolated twice from faeces, and $C$ tropicalis was isolated once.

Finally, case 12 was colonised throughout three hospital admissions mainly by $C$ albicans and $C$ krusei, but the $C$ albicans biotypes conformed to no particular pattern or majority consensus, and two other yeast species, C famata and $S$ cerevisiae, were isolated on rare occasions.

\section{LONGITUDINAL CHANGES IN YEAST FLORA} DURING AND BETWEEN HOSPITAL ADMISSIONS

The results in table 3 showed that even if the complication of changes in $C$ albicans biotypes were disregarded, the yeast microflora showed substantial variations from time to time in some patients while it remained very consistent in others. The full body of isolation data was therefore analysed to determine the incidence and nature of changes in yeast flora within a single hospital admission and between admissions.

For analysis of changes in commensal yeast flora among the study population within one continuous period in the ward, data were analysed for a single hospital admission only for each patient, and the analysis was restricted entirely to oral and faecal yeast isolates. For inclusion in the analysis a patient had to have a minimum of two yeast positive cultures from either oral swabs or faeces during the hospital admission. Data that met these criteria were available for 38 patients with yeast positive oral swabs and for 44 patients with yeast positive faeces (table 4). The consensus biotype results for $C$ albicans isolates were used to avoid overinterpretation of changes in biotype that might have resulted from technical considerations. The period of observation for each patient was calculated on the basis of the period between the first and last yeast positive cultures obtained within the admission, as the analysis was concerned only with species and biotype changes in a yeast positive flora: the data available were insufficient to permit analysis of acquisition and loss of yeast flora of any type.

The results for individual patients in table 3 show how rarely a complete exchange of one yeast species for another occurred within the study population. A change in flora was therefore defined as the
Table 4 Appearance of new Candida species or $C$ albicans biotypes in oral or faecal flora of patients within one hospital admission

\begin{tabular}{lclll}
\hline Sample source & $\begin{array}{l}\text { Duration of } \\
\text { observation* }\end{array}$ & $\begin{array}{l}\text { No of } \\
\text { patients }\end{array}$ & $\begin{array}{l}\text { No (\%) } \\
\text { with new } \\
\text { species }\end{array}$ & $\begin{array}{l}\text { No (\%) } \\
\text { with new } \\
\text { biotype }\end{array}$ \\
\hline Oral cavity & $\begin{array}{l}1-14 \text { days } \\
\text { Stools }\end{array}$ & 18 & $3(17)$ & $4(22)$ \\
& $>14$ days & 20 & $2(10)$ & $2(10)$ \\
& $>14$ days & 20 & $5(25)$ & 0 \\
& $>14$ days & 24 & $19(79)$ & $1(4)$ \\
\hline
\end{tabular}

*Time between first and last yeast positive cultures obtained.

appearance of any yeast species or $C$ albicans biotype different from that or those encountered in the earliest yeast positive sample. On this basis it was evident that changes in the yeast flora occurred significantly more often in faecal samples ( $57 \%$ showed some change) than in oral samples ( $29 \%$ showed some change); $\chi^{2}$ test, $\mathrm{p}<0.02$ (table 4). In faecal samples the changes in flora occurred significantly more often after 14 days of yeast colonisation than within a 14 day period (table 4; $\chi^{2}$ test, $\mathrm{p}<0.01$ ).

Of 49 patient admissions in which the positive oral or faecal sample contained $C$ albicans, a new yeast species was subsequently found in only $11(22 \%)$; in 33 patient admissions where the first yeast isolated was a species other than $C$ albicans, a new species subsequen- 8 tly appeared in $18(55 \%)\left(\chi^{2}\right.$ test, $\left.\mathrm{p}<0.02\right)$. C albicans and $C$ glabrata were the two species which seemed to be acquired most commonly as replacement or: additional yeast flora among the patients studied (table 5). There were no statistical associations between changes in the yeast flora and the nature of antifungal prophylaxis used or the time of species change subsequent to the time of hospital admission.

Twenty five of the patients were admitted to the ward on at least two separate occasions during the study. For 15 of these patients there were at least two yeast positive samples from a single anatomical source within each admission, so that changes in flora between admissions could be assessed. Only three of the 15 patients showed any change in their yeast flora that could be interpreted as a change between admissions; the other 12 retained essentially the same yeast flora, sometimes throughout three or more admissions (table 3). The three flora changes were all of a different nature. One patient whose faecal isolates were $C$ glabrata and $S$ cerevisiae on the first admission consistently carried $C$ albicans type 146 on the second admission (case 9, table 3). One patient from whom only $C$ albicans was isolated from faeces during the first admission had $C$ glabrata present as well as $C$ albicans in faeces during the second admission (case 10 ). Finally, case 12 , with a single oral swab positive for $C$ famata during the first admission, was colonised 
Table 5 Occurrence of yeast species as additional or replacement flora in one hospital admission

\begin{tabular}{lll}
\hline Species & $\begin{array}{l}\text { Incidence in oral } \\
\text { samples }\end{array}$ & $\begin{array}{l}\text { Incidence in faecal } \\
\text { samples (33 patients) }\end{array}$ \\
\hline Calbicans & 2 & 11 \\
C glabrata & 1 & 10 \\
C krusei & 1 & 3 \\
C parapsilosis & 1 & 1 \\
G tropicalis & 0 & 1 \\
S cerevisae & 2 & 4
\end{tabular}

In patients from whom more than one yeast species was isolated subsequent to the first sample, each new species is counted separately.

orally with $C$ krusei and $C$ albicans during the second admission.

\section{Discussion}

As far as we know this study is the first broadly based study of commensal yeast colonisation among patients with haematological disease. Yeast species found among such patients occur in a complex manner and faecal samples are more likely than oral samples to show changes in the yeast species present during a patient's hospital stay.

The spectrum of yeast species isolated overall from these patients can be compared with that found among cancer patients of all types. Kiehn et al found that $C$ albicans accounted for $73 \%$ of oral yeast isolates and $72 \%$ of faecal yeast isolates from their cancer patients. ${ }^{6}$ Tomoda et al found this species in $69 \%$ of faecal isolates from their cancer patients. ${ }^{2}$ The figures for oral and faecal yeast species in our survey showed prevalences of $C$ albicans of $64 \%$ and $45 \%$, respectively, which indicates a lower preponderance of $C$ albicans among the patients with leukaemia than among other cancer patients. Whether this difference is specifically related to haematological disease or to other factors such as the selective pressures of antifungal prophylaxis as used in our patients is not known.

A yeast flora persisted among many patients despite the use of prophylactic, orally administered antifungal drugs. No antifungal agent has yet been shown to be capable of eliminating the commensal yeast flora in patients with leukaemia, although agents such as amphotericin $B$ and ketoconazole seem to reduce the incidence of life threatening systemic Candida infections among this population.?

Both the value and the inadequacies of the biotyping method used to differentiate $C$ albicans strains in the present study have been shown very plainly. Indeed, this is the most substantial study in which $C$ albicans biotyping has been applied blind to a very large number of yeast isolates over a long period of time. The data in table 3 confirm the inherent reliability of the method, as in four of the patients (case 2, 4, 5 and 9) a highly acceptable consistency in biotypes was found over a period of time. Similarly consistent biotyping results were found in other patients who harboured $C$ albicans over long periods but for whom the total number of yeast isolates in the study was fewer than for those included in table 3 . On the other hand, results showing wider variations in biotype data were obtained longitudinally in cases 6,7 , 8,9 , and 10 although even among most of these patients there was a tendency towards a consistent underlying biotype pattern. Results from the Candida biotyping tests used in this study are known to be highly vulnerable to technical performance variables, particularly to variance in inoculum size,$^{38}$ so the use of "consensus" biotype results determined by computer for each patient avoids overinterpretation of apparent changes in $C$ albicans. On this basis it seems that patients with leukaemia change their $C$ albicans biotype less often than they change the species of yeast with which they are colonised. Biotype results cannot be reliably compared between studies, ${ }^{8}$ so no attempt is made here to relate the $C$ albicans biotypes found in this patient group with those in other surveys.

We can only speculate as to why the faecal yeast flora seems to change more often than the oral yeast flora among the patients studied. Because it seems that a site colonised with $C$ albicans is significantly less likely to undergo a change in commensal yeast species than a site harbouring another species, the reduced initial prevalence of $C$ albicans in the faeces of our patients seems to render the lower bowel more prone to colonisation by other yeasts. Certainly, $C$ albicans adheres better than other yeast species to epithelial surfaces,' which may explain the comparative resilience of intestinal sites occupied by $C$ albicans to recolonisation.

The relative consistency of our patients' commensal yeast flora, both within and between admissions to the haematology wards, provides little evidence for cross contamination of the patients with yeasts from the environment, staff, or other patients. Apparent outbreaks of systemic Candida infection have been documented in some intensive care units ${ }^{910}$ and a neonatal special care unit, ${ }^{11}$ though not yet in specialist leukaemia wards. The results of our extensive survey suggest such outbreaks of candidosis result from the chance introduction into these closed units of strains with an unusually high propensity to colonise the patients, and that they do not represent an ever present hazard from an inherent tendency of "ward" strains of Candida to cross infect patients readily.

This study was supported by a grant from the Wellcome Trust. We are grateful to B K Basatia for 
skilled technical assistance and L Gandhi for help with the data collection.

\section{References}

1 Odds FC. Candida and candidosis 2nd ed. London: BaillièreTindall, 1988.

2 Tomoda T, Nakano Y, Kageyama T. Variations in intestinal Candida populations in patients receiving antileukaemic therapy. Bull Osaka Med School 1984;30:14-8.

3 Odds FC, Abbott AB. A simple system for the presumptive identification of Candida albicans and differentiation of strains within the species. Sabouraudia 1980;18:301-18.

4 Odds FC, Abbott AB. Modification and extension of tests for differentiation of Candida species and strains. Sabouraudia 1983;21:79-81.

5 Land GA, Vinton AC, Adcock GB, Hopkins JM. Improved auxanographic method for yeast assimilations: a comparison with other approaches. J Clin Microbiol 1975;2:206-17.

6. Kiehn TE, Edwards FF, Armstrong D. The prevalence of yeasts in clinical specimens from cancer patients. Am J Clin Pathol 1980;73:518-21.

7 Hann IM, Prentice HG, Corringham R, et al. Ketoconazole versus nystatin and amphotericin $\mathbf{B}$ for fungal prophylaxis in severely immunocompromised patients. Lancet 1982; i:826-9.

8 Odds FC, Auger P, Krogh P, Neely AN, Segal E. Biotyping of Candida albicans - results of an international collaborative survey. J Clin Microbiol 1989;27:1506-9.

9 Burnie JP, Odds FC, Lee W, Webster C, Williams JD. Outbreak of systemic Candida albicans in intensive care unit caused by crossinfection. Br Med J 1985;290:746-8.

10 Matthews R, Burnie JP. Assessment of DNA fingerprinting for rapid identification of outbreaks of systemic candidiasis. $\mathrm{Br}$ Med J 1989;298:354-7.

11 Phelps M, Ayliffe GAJ, Babb JR. An outbreak of candidiasis in a special care baby unit: the use of a resistogram typing method. J Hosp Infect 1986;7:13-20.

Requests for reprints to: Dr.F C Odds, Laboratory of Bacteriology and Mycology, Janssen Research Foundation, Turnhoutseweg 30, B-2340, Beerse, Belgium. 